\title{
Factors influencing gastrointestinal tract and microbiota immune interaction in preterm infants
}

\author{
María Carmen Collado', María Cernada ${ }^{2,3}$, Josef Neu${ }^{4}$, Gaspar Pérez-Martínez', María Gormaz ${ }^{3}$ and Máximo Vento ${ }^{2,3}$
}

The role of microbial colonization is indispensable for keeping a balanced immune response in life. However, the events that regulate the establishment of the microbiota, their timing, and the way in which they interact with the host are not yet fully understood. Factors such as gestational age, mode of delivery, environment, hygienic measures, and diet influence the establishment of microbiota in the perinatal period. Environmental microbes constitute the most important group of exogenous stimuli in this critical time frame. However, the settlement of a stable gut microbiota in preterm infants is delayed compared to term infants. Preterm infants have an immature gastrointestinal tract and immune system which predisposes to infectious morbidity. Neonatal microbial dynamics and alterations in early gut microbiota may precede and/or predispose to diseases such as necrotizing enterocolitis (NEC), late-onset sepsis or others. During this critical period, nutrition is the principal contributor for immunological and metabolic development, and microbiological programming. Breast milk is a known source of molecules that act synergistically to protect the gut barrier and enhance the maturation of the gut-related immune response. Host-microbe interactions in preterm infants and the protective role of diet focused on breast milk impact are beginning to be unveiled.

C urrent evidence supports the role of microbiota in promoting and maintaining a balanced immune response and the establishment of the gut barrier in the immediate postnatal life. The human body harbors a dynamic and complex microbial population, which includes around 500-1,000 different species. In the perinatal period, neonates are exposed not only to a vast microbial diversity but also to a variety of organisms such as viruses, fungi and parasites. After weaning, the infant's gut is colonized by a rapidly diversifying microbiota that leads to an adult-like pattern of intestinal flora. The composition of the neonatal microbiota is influenced by numerous perinatal factors such as mode of delivery, environment, hygienic measures, antibiotic treatment, and breastfeeding practices (1). Adverse circumstances affecting the establishment of a normal microbiota may result in failure in the development of a balanced immune response but also have a significant impact on the intestinal mucosal barrier function and intestinal maturation. Furthermore, these disturbances may predispose to specific diseases later in life. Early host-microbe interactions may provide a signal for immune system development and maturation (2).

The "Early programming" theory is based upon the fact that environmental exposures including nutritional during the perinatal period can induce permanent changes in many physiological processes. Postnatal diet mainly breastfeeding practices are therefore critical for the ongoing developmental maturation of many organ systems and optimal physiological functions The effects of early programming extend into adulthood and are linked to the risk of acquiring specific diseases in adult life (3-5).

\section{PREMATURITY AND MICROBIOTA}

In the last decades, mortality of very preterm infants has been substantially reduced. Improvement in the survival rates has been attributed to a series of factors such as regionalization, generalization of antenatal steroids, new modalities of mechanical ventilation, and the use of surfactant replacement therapy (6). However, this has not always been paralleled by a simultaneous decrease in morbidity. The prevalence of serious complications such as retinopathy of prematurity, bronchopulmonary dysplasia, or periventricular leukomalacia has remained practically unchanged especially in the very preterm infants ( $<32 \mathrm{wk}$ gestation) (7). Of note, mortality as a consequence of neonatal septicemia has been reported to be around $18 \%$ of all neonatal deaths. Interestingly, $~ 50 \%$ of these deaths occur in the first week of life, and the remaining $50 \%$ are nosocomial infections acquired during prolonged hospitalization (8).

The role of microbiota in preterm birth and the consequences of prematurity upon postnatal microbiome development are emerging fields of discussion. The exact mechanisms responsible for preterm birth are multifactorial and yet not completely unveiled (Table 1). However, the link between infection and prematurity has been well established. In addition to the traditionally accepted ascending infection from the 
lower genital tract recent research suggests that other forms of bacterial spreading could be responsible for preterm delivery (9). Interestingly, studies by Madianos et al. (10) and Aargard et al. (11) have shown resemblances between fetal and neonatal bacterial colonization and maternal oral cavity and placental flora strongly suggesting a hematogenous dissemination in the perinatal period.

The interaction of preterm infants' altered gut microbiota with an immature immunologic intestinal response triggers proinflammatory and counter-inflammatory cytokine response (12). These factors are extremely important for preterm infants because they influence the pattern of bacterial gut colonization, which is different to that in the healthy full-term infant (Figure 1). Briefly, preterm infants showed retarded Bifidobacterium- microbiota colonization, a high prevalence of Staphylococcus, Enterobacteriaceae, Enterococcaceae and other lactic acid bacteria as the genus Lactobacillus and Weissella in a low-diversity bacterial ecosystem (13-15). In preterm birth a negative correlation with gestational age and a tendency toward a higher inflammatory response has been associated with higher presence of Enterobacter, Enterococcus, Lactobacillus, Photorhabdus, and Tannerella spp. (17). Microbial colonization of meconium and feces of preterm infants analyzed during the first months of life using traditional culture-based methods and molecular techniques to analyze bacterial diversity revealed that Firmicutes was the predominant phylum in meconium while Proteobacteria was present in neonatal fecal samples. Interestingly, the Proteobacteria phylum may increase the inflammatory response in the preterm intestine, and it has been speculated that exposure to low quantities of lipopolysaccharides, flagellin, or other Gram-negative substances may interact with the intestinal Toll-like receptors (TLR) and confer "tolerance" to further inflammatory stimuli (18). In a recent study, it was shown that early Proteobacteria colonization might cause an exacerbated immune response, which may impact on the intestinal barrier enhancing bacterial translocation and the risk to develop sepsis, necrotizing enterocolitis (NEC) and other inflammatory problems (19-21). The concept of bacterial translocation and gut-originated sepsis and NEC as a cause of systemic infectious complications has emerged over the last years, although the exact clinical relevance of these phenomena continues to be debated (21).

\section{GASTROINTESTINAL TRACT IMMUNE SYSTEM AND MICROBIOME INTERACTION IN PRETERM INFANTS}

At birth, the adaptive immune system is quite rudimentary and untrained. Hence, normal term newborns highly rely on trans-placental passage of mother's IgG during the last 3 mo of gestation for control of pathogens showing a remarkable activation of $\mathrm{T}$ cells $(22,23)$. Furthermore, the immune response to gut pathogens in term newborn infants still requires protein components from mother's milk to efficiently fulfill TLR binding and signal transduction (24). Innate immune functions in the fetus are ontologically regulated through gestation; hence, developmental events may give rise to differences in the innate immune response of the neonate, and particularly in premature newborns, putting them at high risk of developing severe infections. A high proportion of Th1-polarizing cytokines during pregnancy has been related to a high probability of abortion (25). Therefore, in the fetus, expression of Th2 cytokines

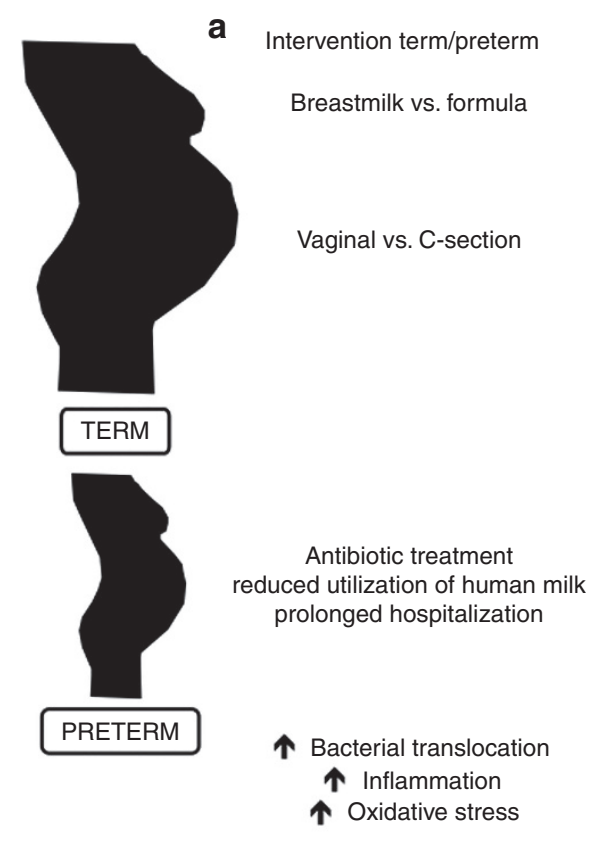

b

b Microbiome impact

个 Bifidobacterium spp

$\downarrow$ Enterobacteriaceae

个 Lactobacillus, Prevotella and Sneathia

(maternal vaginal and intestinal microbiota)

$\downarrow$ Staphylococcus, Propionibacterium and Corynebacterium (skin and oral microbiota, environmental bacteria)

Delayed bacteroides colonization

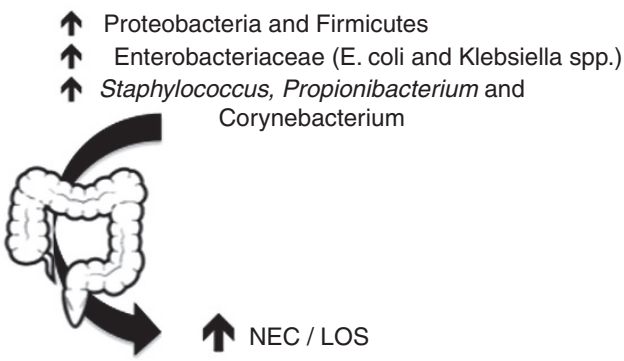

Figure 1. Factors influencing preterm infants pattern of bacterial gut colonization. (a) Perinatal factors influencing gut microbiota composition in term and preterm infants (intervention term/preterm). (b) The influences of perinatal factors upon microbiome composition in term and preterm infants. In preterm infants, antibiotic treatment, reduces utilization of human milk and prolonged hospitalization prompts the development of specific microbial strains that cause bacterial translocation, inflammation, and oxidative stress thus contributing to the development of necrotizing enterocolitis and/or late-onset sepsis. 
Table 1. Contributing causes to preterm delivery

\begin{tabular}{ll}
\hline $\begin{array}{l}\text { Maternal conditions } \\
\text { Preterm C-section }\end{array}$ & Pre-eclampsia, fetal distress \\
$\begin{array}{l}\text { Assisted reproductive } \\
\text { techniques }\end{array}$ & Multiple gestations, older maternal age \\
$\begin{array}{l}\text { Surgery performed to the } \\
\text { mother }\end{array}$ & \\
Social stress and/or race & $\begin{array}{l}\text { Low maternal age, limited maternal } \\
\text { education, poverty, unmarried status } \\
\text { Chorioamnionitis, inflammation of } \\
\text { membranes }\end{array}$ \\
Infection and/or inflammation & \\
$\begin{array}{l}\text { Genetic and epigenetic } \\
\text { (environmental) factors }\end{array}$ & \\
\hline Data taken from ref. 9. &
\end{tabular}

may be the strategy to avoid negative reactions. However, premature birth renders the newborn more susceptible to infections (22).

Nevertheless, preterm infants have a substantially reduced trans-placental transfer of maternal antibodies and an immature innate immune system. Both these circumstances facilitate the preterm to acquire infections $(23,24)$. Shorter gestational duration is associated with reduced proportions of peripheral blood lymphocytes (26), proinflammatory cytokine response and secretion of antibacterial peptides, smaller counts of phagocytic peripheral monocytes, and reduced leukocyte endothelial transmigration $(27,28)$. The reduced synthesis of proinflammatory cytokines, such as IL-1 $\beta$, IL-6, TNF- $\alpha$, etc., in response to bacterial endotoxin lipopolysaccharide binding to TLR4 does not depend on the low expression of TLR4 and CD14 but to a yet unknown factor downstream in the signal transduction pathway $(29,30)$. Transmigration of neutrophils in innate immunity is an essential process for immune cell recruitment toward inflamed tissues. It is a highly regulated process that involves a series of adhesion steps to specific molecules comprising an initial "rolling" of neutrophils on the endothelial surface, followed by firm adhesion, and finally transmigration and exit from the blood vessel. Therefore, this process is favored by Th1-polarizing cytokines (proinflammatory); however, the fetus follows a Th2-polarization strategy that affects the efficiency of transmigration (22). Finally, there is a likely reduction in phagocytic activity by monocytes, with respect to term newborns $(24,29)$, but also of gut dendritic cells (30). These cells ultimately eliminate bacteria and therefore constitute an essential component of the innate immune system; however, they also intervene in the process of antigen presentation, which constitutes the initial step in the adaptive immune response.

Abnormal patterns of colonizing gut bacteria in infants with an immature host innate and adaptive immune system will accelerate de advent of infectious diseases, particularly when catheters and enteric feeding facilitate pathogenic invasion. The remarkable importance of maintenance of immune homeostasis in preterm infants derives from the fact that, although clinical and pharmacological approaches have decreased mortality of infected preterm infants, these processes cause a high proinflammatory and pro-oxidant stress that inevitably leads to irreversible damage to vital organs, including brain and intestine that often results in neurodevelopment impairment (31) and gut bacteria over-reactivity that may lead to IBD (32).

\section{SEPSIS AND MICROBIOME}

Sepsis remains one of the most common causes of neonatal morbidity and mortality in the preterm population $(8,33)$. Early-onset sepsis occurs in $1.5-1.9 \%$ of very-low-birth weight and late-onset sepsis (LOS) in 20\%, with mortality altogether approaching 18\% (8) and a higher risk of cerebral palsy in preterm infants (33). Gram-negative organisms are the most prevalent microbes in LOS, while Gram-positive organisms especially acquired during first days of life and also, Coagulase-negative Staphylococcus are the most common microbes followed by specific Gram-negative bacteria in LOS (8). However, it has been shown that a dysbiosis in preterm microbiota composition but not an increased prevalence of potential pathogens is associated with sepsis (19). Thus, the presence of Enterobacter and Staphylococcus spp. has been associated with NEC and sepsis, respectively (34). It has been also described that lower bacterial diversity present in meconium samples from preterm infants is related to higher risk of sepsis (35). Our results in very-low-birth-weight preterm twins showed higher levels of Enterobacteriaceae family and lower levels of Bifidobacterium spp in the sepsis neonates as those observed in their healthy twin controls. By pyrosequencing, we also found a high presence of Proteobacteria phylum (Enterobacteriaceae family) in septic infants. Principal coordinate analysis (PCoA) showed differences between sepsis and control groups although microbial profiles were twin-pair and neonate-dependent.

\section{NEC AND MICROBIOME}

NEC has become the one of the most severe and dreaded diseases seen in neonatal intensive care (36). In North America, it affects $\sim 7 \%$ of babies weighing between $500-1,500 \mathrm{~g}$ and $\sim 20-30 \%$ of these babies die of this disease (37). Sequels are not limited to the gastrointestinal system, where short gut is a common problem, but severe neurodevelopmental delays are commonly seen especially after NEC that requires surgery (38). The initiation of early enteral feeding in preterm infants favors an earlier achievement of full enteral feeding and does not increase the risk of NEC; however, the optimal advance of enteral feedings remains a debatable question that ongoing studies intend to clarify (39).

The pathophysiology of the most common form of this disease seen in preterm infants has remained elusive; however, several factors predisposing to the development of this disease have been identified. Functional immaturity of the gut likely plays a role (36). The fact that the disease often occurs in clusters has suggested a microbial etiology. As of yet, no single microbe has been found that is causative, but an inappropriate colonization prior to the development of the disease has been suggested (40). 


\section{Preterm microbiota | REVIOW}

Studies of the microbial ecology prior to the development of NEC using sequencing technology show differences in those babies who develop the disease vs. matched controls $(40,41)$. Preliminary studies on fecal microbiota from unaffected preterm infants and from infants with NEC both prior to and during NEC showed an association between intestinal microbial species and NEC. A matched analysis of predominant phyla prior to the development of the disease shows that Proteobacteria or the relative proportion of Proteobacteria with the other phyla may be involved. The Proteobacteria phylum contains numerous Gram-negative genera such as Klebsiella and Escherichia coli, which may be pathogenic $(42,43)$.

Promising approaches to prevent NEC are directed toward the inhibition of TLR4. In experimental models, the molecule C34, a 2-acetamidopyranoside (MW389) (44) and amniotic fluid (45) have reduced the incidence of experimental NEC. Epithelial growth factor (EGF) (46) and hepatocyte growth factors (HGF) (47) were found to be the mediators of amniotic fluid-derived protection. An improved understanding of the factors influencing the development of the microbiota or that modulate its composition may offer new strategies, tools, and opportunities for preterm interventions that reduce the risk of specific diseases as NEC.

POTENTIAL TOOLS TO MODULATE PRETERM MICROBIOME Among the different strategies employed to reduce the incidence and/or severity of NEC and prevent LOS in preterm infants, the use of human breast milk (HM) and/or supplementation with pro-and/or-prebiotics have rendered effective results (48-50). In the following paragraphs, we will expand on these strategies.

\section{HUMAN BREAST MILK AS GOLD STANDARD DIET FOR PRETERM}

HM is considered the gold standard for infant nutrition and constitutes the main postnatal link between mother and infant. HM contains bioactive components that directly influence the developing infant and shape the development of the intestinal microbiota. Beyond the nutritional composition, it contains several immune-related substances such as regulatory cytokines and growth factors, which are considered protective and stimulate the immune system with a positive impact on health. In addition, it contains other active nonspecific factors, such as lysozyme, lactoferrin, oligosaccharides and also, microorganisms, which also contribute to enhance anti-infective and immune-modulatory properties (51). The implication of HM in both prevention and treatment of NEC has long been recognized; however the specific compounds responsible for these beneficial effects are yet unknown. Hence, several recent studies have reported that lactoferrin might be able to minimize LOS and also NEC $(49,52)$. The ability of preterm infants to respond to pathogens, as the reported incidence of NEC and LOS indicates, inversely correlates to the gestational age (53), and enteral formula diets when coupled to parenteral nutrition predispose to NEC, while progressive nutrition with colostrum and mother's milk show a protective effect (50).
Perhaps the increased amount of polyamines in breast milk known to have a protective effect on beneficial microbiota constitutes a natural contribution of mothers that give birth preterm $(54,55)$. Another interesting source of protection is related to the immaturity of the antioxidant defense system of preterm infants (55). Oxidative stress-derived free radicals are relevant contributing factors to a generalized inflammatory response, which sets the basis for organ/system damage (56). Interestingly, it has been shown that feeding with preterm human milk is protective against hydroxyl radical aggression as compared to formula feeding. Hence, preterm babies fed own mother's milk eliminated significantly less biomarkers of oxidative damage to proteins and DNA in the urine as compared to paired formula fed preterm infants (58).

Fresh own mother's milk provided directly from the breast is the gold standard as all its biologically active components are preserved. Preterm infants require tube feeding and are either fed fresh expressed, frozen, and sometimes pasteurized donor human milk. Milk storage and processing affects bioactive compounds, but still donor pasteurized human milk appears to provide protection from NEC when compared to formula $(59,60)$.

\section{MICROBES TO MODULATE PRETERM MICROBIAL COMPOSITION}

Preterm infants are endowed with lower microbial diversity compared to term neonates. The use of probiotics could modulate towards a similar microbial community to those observed in term infant or adult gastrointestinal microbiome and ameliorate microbiome status of preterm infants. (61). Studies that promote exogenous supplementation with probiotics to preterm infants are based on the hypothesis that microbiota of preterm infants can be modulated by exogenous bacteria which results in an improvement in clinical outcomes. Probiotic bacteria are able to exert beneficial activity on intestinal epithelial cells, microbiota modulation, and immune system response through different mechanisms of action which include: (i) competitive exclusion, inhibition, and displacement of potential pathogenic organisms adhesion and also, nutrient competition; (ii) production of antimicrobial compounds; (iii) improvement of barrier function; (iv) modulation of immune response; and (v) reduction of inflammation by interacting of NF- $\kappa B$ pathways (62).

The prevention of NEC in extremely low-birth-weight (ELBW) infants derived from the prophylactic use of probiotics has not yet been clearly established. In the most recent Cochrane review and meta-analysis on the prevention of NEC using prophylactic probiotics a total of 24 randomized and quasi-randomized studies were included. Prevention with probiotics, which contained either Lactobacillus spp. alone or in combination with Bifidobacterium spp. was analyzed. Results of the meta-analysis suggested that probiotic supplementation may have some positive effects in relation with overall mortality and NEC, but did not influence the incidence of nosocomial sepsis. In addition, no side effects due to probiotic treatment were reported (48). However, it should be underscored that 
most of the studies included in this meta-analysis were not blinded, had a high variability in the enrolment criteria, in the feeding regimes, in the probiotic composition and dosing, and in the basal incidence of NEC, and treatment regimes. Moreover, ELBW infants were under-represented or not beneficial effects found in this specific population that represents the highest at-risk patients for NEC and/or septicemia. At present, there is insufficient evidence to recommend routine prophylactic use of probiotics to prevent NEC in ELBW infants. Moreover, there are still many unanswered questions regarding the use of probiotics in the clinical setting before its use gets generalized. Hence, identification of the appropriate probiotic-strain, rigorous quality control of manufacturing of this probiotic as a pharmaceutical agent rather than a food, dose, timing and length of treatment, effect on highest risk population or in exclusively breast fed infants, remain unanswered. Potential long-term consequences such as modification of host gene expression, influence on ongoing bacterial colonization, immune-modulation, or antibiotic resistance have not been explored. Under these circumstances, it has been recently proposed the need for a high-quality NEC prevention clinical trial using probiotics in at-risk ELBW infants fulfilling the stringent guidelines of the International Conference of Harmonisation for Good Clinical Practice (www.ich.org) (63).

Interestingly, inactivated probiotics may also play a role modulating excessive inflammatory stimuli. Thus, preterm infants treated with inactivated probiotics showed a decreased incidence of NEC as compared to the control babies (64)

Further, large clinical trials are required to answer these issues and to support and push the development of new probiotic products, which would benefit preterm population.

\section{PREBIOTICS}

Prebiotics are nondigestible food ingredients such as oligosaccharides that are beneficial because they selectively stimulate the growth and/or biologic activities of intestinal bacteria in the colon thus contributing to ameliorate the host's health status. Prebiotics promote the growth of nonpathogenic organisms such as bifidobacteria. Only few studies have been performed in the clinical setting. The most recent updated review and meta-analysis which included a total of 7 studies and 417 preterm babies enrolled, concluded that the use of oligosaccharides enhanced beneficial bacterial growth but did not reduce the incidence of NEC, LOS, or modify time to achievement of full enteral feedings (64).

\section{CONCLUSIONS}

To conclude, perinatal and early postnatal time represent the most critical periods for the establishment of the microbiota, which exerts a key role in the establishment of the gut barrier and as an immune-modulator. Numerous perinatal factors influence neonatal microbiota such as mode of delivery, environment, hygienic measures, antibiotic use, and breastfeeding practices. Preterm infants altered gut microbiota interaction with an immature immunologic intestinal response triggers proinflammatory and counter-inflammatory cytokine response.
Breast milk is the gold standard for infant nutrition and influences the development of intestinal microbiota and immune system through its bioactive components. Probiotics may be promising in the prevention of NEC in certain populations of preterm infants. Future research should aim to explore appropriate treatment regimes, strain-specific effects on sub-selected populations and long term effects of probiotic administration.

\section{STATEMENT OF FINANCIAL SUPPORT}

M.C. acknowledges a "Rio Hortega" Research Fellowship Grant (CM13/0017) and M.V. acknowledges grants PI1 1/0313 and RD12/0026/0012 (Red SAMID) from the Instituto Carlos III (Spanish Ministry of Economy and Competitivity). M.C.C. and G.P-M. were supported by the grant AGL2013-47420-R from the Spanish Ministry of Science and Innovation.

\section{REFERENCES}

1. Collado MC, Cernada M, Baüerl C, Vento M, Pérez-Martínez G. Microbial ecology and host-microbiota interactions during early life stages. Gut Microbes 2012;3:352-65.

2. Hollister EB, Gao C, Versalovic J. Compositional and functional features of the gastrointestinal microbiome and their effects on human health. Gastroenterology 2014;146:1449-58.

3. Canani RB, Costanzo MD, Leone L, et al. Epigenetic mechanisms elicited by nutrition in early life. Nutr Res Rev 2011;24:198-205.

4. Nauta AJ, Ben Amor K, Knol J, Garssen J, van der Beek EM. Relevance of preand postnatal nutrition to development and interplay between the microbiota and metabolic and immune systems. Am J Clin Nutr 2013;98:586S-93S.

5. Madan JC, Farzan SF, Hibberd PL, Karagas MR. Normal neonatal microbiome variation in relation to environmental factors, infection and allergy. Curr Opin Pediatr 2012;24:753-9.

6. Vento M, Moro M, Escrig R, et al. Preterm resuscitation with low oxygen causes less oxidative stress, inflammation, and chronic lung disease. Pediatrics 2009;124:e439-49.

7. Fanaroff AA, Stoll BJ, Wright LL, et al.; NICHD Neonatal Research Network. Trends in neonatal morbidity and mortality for very low birthweight infants. Am J Obstet Gynecol 2007;196:147.e1-8.

8. Stoll BJ, Hansen N, Fanaroff AA, et al. Late-onset sepsis in very low birth weight neonates: the experience of the NICHD Neonatal Research Network. Pediatrics 2002;110(2 Pt 1):285-91.

9. Muglia LJ, Katz M. The enigma of spontaneous preterm birth. N Engl J Med 2010;362:529-35.

10. Madianos PN, Bobetsis YA, Offenbacher S. Adverse pregnancy outcomes (APOs) and periodontal disease: pathogenic mechanisms. J Periodontol 2013;84(4 Suppl):S170-80.

11. Aagaard K, Ma J, Antony KM et al. The placenta harbors a unique microbiome. Sci Transl Med 2014;6:237.ra65.

12. Torrazza RM, Neu J. The altered gut microbiome and necrotizing enterocolitis. Clin Perinatol 2013;40:93-108.

13. Jacquot A, Neveu D, Aujoulat F, et al. Dynamics and clinical evolution of bacterial gut microflora in extremely premature patients. J Pediatr 2011;158:390-6.

14. Arboleya S, Binetti A, Salazar N, et al. Establishment and development of intestinal microbiota in preterm neonates. FEMS Microbiol Ecol 2012;79:763-72.

15. Arboleya S, Solís G, Fernández N, de los Reyes-Gavilán CG, Gueimonde M. Facultative to strict anaerobes ratio in the preterm infant microbiota: a target for intervention? Gut Microbes 2012;3:583-8.

16. LaTuga MS, Ellis JC, Cotton CM, et al. Beyond bacteria: a study of the enteric microbial consortium in extremely low birth weight infants. PLoS One 2011;6:e27858.

17. Ardissone AN, de la Cruz DM, Davis-Richardson AG, et al. Meconium microbiome analysis identifies bacteria correlated with premature birth. PLoS One 2014;9:e90784.

18. Moles L, Gómez M, Heilig H, et al. Bacterial diversity in meconium of preterm neonates and evolution of their fecal microbiota during the first month of life. PLoS One 2013;8:e66986. 


\section{Preterm microbiota | ReView}

19. Mai V, Torrazza RM, Ukhanova M, et al. Distortions in development of intestinal microbiota associated with late onset sepsis in preterm infants. PLoS One 2013;8:e52876.

20. Sherman MP. New concepts of microbial translocation in the neonatal intestine: mechanisms and prevention. Clin Perinatol 2010;37:565-79.

21. Deitch EA. Gut-origin sepsis: evolution of a concept. Surgeon 2012;10: 350-6.

22. Nussbaum C, Sperandio M. Innate immune cell recruitment in the fetus and neonate. J Reprod Immunol 2011;90:74-81.

23. Luciano AA, Yu H, Jackson LW, Wolfe LA, Bernstein HB. Preterm labor and chorioamnionitis are associated with neonatal $\mathrm{T}$ cell activation. PLoS One 2011;6:e16698.

24. Goldman AS. The immune system in human milk and the developing infant. Breastfeed Med 2007;2:195-204.

25. Makhseed M, Raghupathy R, Azizieh F, Omu A, Al-Shamali E, Ashkanani $\mathrm{L}$. Th1 and Th2 cytokine profiles in recurrent aborters with successful pregnancy and with subsequent abortions. Hum Reprod 2001;16:2219-26.

26. Milcic TL. The complete blood count. Neonatal Netw 2010;29:109-15.

27. Sharma AA, Jen R, Butler A, Lavoie PM. The developing human preterm neonatal immune system: a case for more research in this area. Clin Immunol 2012;145:61-8.

28. Strunk T, Currie A, Richmond P, Simmer K, Burgner D. Innate immunity in human newborn infants: prematurity means more than immaturity. J Matern Fetal Neonatal Med 2011;24:25-31.

29. Strunk T, Prosser A, Levy O, et al. Responsiveness of human monocytes to the commensal bacterium Staphylococcus epidermidis develops late in gestation. Pediatr Res 2012;72:10-8.

30. Currie AJ, Curtis S, Strunk T, et al. Preterm infants have deficient monocyte and lymphocyte cytokine responses to group B streptococcus. Infect Immun 2011;79:1588-96.

31. Walker JC, Smolders MA, Gemen EF, Antonius TA, Leuvenink J, de Vries E. Development of lymphocyte subpopulations in preterm infants. Scand J Immunol 2011;73:53-8.

32. Holloway JA, Thornton CA, Diaper ND, Howe DT, Warner JO. Phenotypic analysis of circulating dendritic cells during the second half of human gestation. Pediatr Allergy Immunol 2009;20:119-25.

33. Stoll BJ, Hansen NI, Adams-Chapman I, et al.; National Institute of Child Health and Human Development Neonatal Research Network. Neurodevelopmental and growth impairment among extremely low-birth-weight infants with neonatal infection. JAMA 2004;292:2357-65.

34. Stewart CJ, Marrs EC, Magorrian S, et al. The preterm gut microbiota: changes associated with necrotizing enterocolitis and infection. Acta Paediatr 2012;101:1121-7.

35. Madan JC, Salari RC, Saxena D, et al. Gut microbial colonisation in premature neonates predicts neonatal sepsis. Arch Dis Child Fetal Neonatal Ed 2012;97:F456-62.

36. Neu J. Necrotizing enterocolitis. World Rev Nutr Diet 2014;110:253-63.

37. Holman RC, Stoll BJ, Curns AT, Yorita KL, Steiner CA, Schonberger LB. Necrotising enterocolitis hospitalisations among neonates in the United States. Paediatr Perinat Epidemiol 2006;20:498-506.

38. Martin CR, Dammann O, Allred EN, et al. Neurodevelopment of extremely preterm infants who had necrotizing enterocolitis with or without late bacteremia. J Pediatr 2010;157:751-6.el.

39. Leaf A. Introducing feeds in the high risk preterm infant. Semin Fetal Neonatal Med 2013;18:150-4.

40. Claud EC, Walker WA. Hypothesis: inappropriate colonization of the premature intestine can cause neonatal necrotizing enterocolitis. FASEB J 2001;15:1398-403.

41. Torrazza RM, Ukhanova M, Wang X, et al. Intestinal microbial ecology and environmental factors affecting necrotizing enterocolitis. PLoS One 2013;8:e83304.

42. Stewart CJ, Marrs EC, Nelson A, et al. Development of the preterm gut microbiome in twins at risk of necrotising enterocolitis and sepsis. PLoS One 2013;8:e73465.

43. Morrow AL, Lagomarcino AJ, Schibler KR, et al. Early microbial and metabolomic signatures predict later onset of necrotizing enterocolitis in preterm infants. Microbiome 2013;1:13.
44. Neal MD, Jia H, Eyer B, et al. Discovery and validation of a new class of small molecule Toll-like receptor 4 (TLR4) inhibitors. PLoS One 2013;8:e65779.

45. Good M, Siggers RH, Sodhi CP, et al. Amniotic fluid inhibits Toll-like receptor 4 signaling in the fetal and neonatal intestinal epithelium. Proc Natl Acad Sci USA 2012;109:11330-5.

46. Siggers J, Ostergaard MV, Siggers RH, et al. Postnatal amniotic fluid intake reduces gut inflammatory responses and necrotizing enterocolitis in preterm neonates. Am J Physiol Gastrointest Liver Physiol 2013;304:G864-75.

47. Jain SK, Baggerman EW, Mohankumar K, et al. Amniotic fluid-borne hepatocyte growth factor protects rat pups against experimental necrotizing enterocolitis. Am J Physiol Gastrointest Liver Physiol 2014;306: G361-9.

48. AlFaleh K, Anabrees J. Probiotics for prevention of necrotizing enterocolitis in preterm infants. Cochrane Database Syst Rev 2014;4:CD005496.

49. Manzoni P, Meyer M, Stolfi I, et al. Bovine lactoferrin supplementation for prevention of necrotizing enterocolitis in very-low-birth-weight neonates: a randomized clinical trial. Early Hum Dev 2014;90 Suppl 1:S60-5.

50. Cristofalo EA, Schanler RJ, Blanco CL, et al. Randomized trial of exclusive human milk versus preterm formula diets in extremely premature infants. J Pediatr 2013;163:1592-1595.e1.

51. Walker A. Breast milk as the gold standard for protective nutrients. J Pediatr 2010;156(2 Suppl):S3-7.

52. Akin IM, Atasay B, Dogu F, et al. Oral lactoferrin to prevent nosocomial sepsis and necrotizing enterocolitis of premature neonates and effect on T-regulatory cells. Am J Perinatol 2014;31:1111-20.

53. Stoll BJ, Hansen NI, Bell EF, et al.; Eunice Kennedy Shriver National Institute of Child Health and Human Development Neonatal Research Network. Neonatal outcomes of extremely preterm infants from the NICHD Neonatal Research Network. Pediatrics 2010;126:443-56.

54. Plaza-Zamora J, Sabater-Molina M, Rodríguez-Palmero M, et al. Polyamines in human breast milk for preterm and term infants. Br J Nutr 2013;110:524-8.

55. Gómez-Gallego C, Collado MC, Pérez G, et al. Resembling breast milk: influence of polyamine-supplemented formula on neonatal BALB/ cOlaHsd mouse microbiota. Br J Nutr 2014;111:1050-8.

56. Vento M, Aguar M, Escobar J, et al. Antenatal steroids and antioxidant enzyme activity in preterm infants: influence of gender and timing. Antioxid Redox Signal 2009;11:2945-55.

57. Vento M. Oxygen supplementation in the neonatal period: changing the paradigm. Neonatology 2014;105:323-31.

58. Ledo A, Arduini A, Asensi MA, et al. Human milk enhances antioxidant defenses against hydroxyl radical aggression in preterm infants. Am J Clin Nutr 2009;89:210-5.

59. Chang JC, Chen CH, Fang LJ, Tsai CR, Chang YC, Wang TM. Influence of prolonged storage process, pasteurization, and heat treatment on biologically-active human milk proteins. Pediatr Neonatol 2013; 54:360-6.

60. Quigley MA, Henderson G, Anthony MY, McGuire W. Formula milk versus donor breast milk for feeding preterm or low birth weight infants. Cochrane Database Syst Rev 2007;4:CD002971.

61. Neu J, Caicedo R. Probiotics: protecting the intestinal ecosystem? J Pediatr 2005;147:143-6.

62. Collado MC, Isolauri E, Salminen S, Sanz Y. The impact of probiotic on gut health. Curr Drug Metab 2009;10:68-78.

63. Abrahamsson TR, Rautava S, Moore AM, Neu J, Sherman PM. The time for a confirmative necrotizing enterocolitis probiotics prevention trial in the extremely low birth weight infant in North America is now! J Pediatr 2014;165:389-94.

64. Awad H, Mokhtar H, Imam SS, Gad GI, Hafez H, Aboushady N. Comparison between killed and living probiotic usage versus placebo for the prevention of necrotizing enterocolitis and sepsis in neonates. Pak J Biol Sci 2010;13:253-62.

65. Srinivasjois R, Rao S, Patole S. Prebiotic supplementation in preterm neonates: updated systematic review and meta-analysis of randomised controlled trials. Clin Nutr 2013;32:958-65. 